\title{
Semiclassical theory for transmission through open billiards: Convergence towards quantum transport
}

\author{
Ludger Wirtz, Christoph Stampfer, Stefan Rotter, and Joachim Burgdörfer \\ Institute for Theoretical Physics, Vienna University of Technology, A-1040 Vienna, Austria
}

(Received 6 June 2002; published 13 January 2003)

\begin{abstract}
We present a semiclassical theory for transmission through open quantum billiards which converges towards quantum transport. The transmission amplitude can be expressed as a sum over all classical paths and pseudopaths which consist of classical path segments joined by "kinks," i.e., diffractive scattering at lead mouths. For a rectangular billiard we show numerically that the sum over all such paths with a given number of kinks $K$ converges to the quantum transmission amplitude as $K \rightarrow \infty$. Unitarity of the semiclassical theory is restored as $K$ approaches infinity. Moreover, we find excellent agreement with the quantum path-length power spectrum up to very long path length.
\end{abstract}

DOI: 10.1103/PhysRevE.67.016206

PACS number(s): 05.45.Mt, 73.23.Ad, 73.50.Bk

\section{INTRODUCTION}

The aim of semiclassical theory is to bridge the gap between quantum mechanics and its classical limit. Generically, probability amplitudes are calculated by summing over classical paths, each of which carries an amplitude and a phase $[1,2]$. Such an approach facilitates an intuitive understanding of basic features of quantum mechanics such as "quantum interference" and allows quantitative calculations in the regime of high energies, i.e., short wavelength $\lambda \rightarrow 0$, where full quantum calculations may become impractical. Moreover, semiclassical theory plays an important role in elucidating the signatures of classical chaos in quantum systems whose classical counterpart is chaotic [1]. Ballistic transport through billiards has become a popular prototype example [3-13]: All paths that connect the entrance lead (or injection quantum wire) with the exit lead (or emission quantum wire) contribute to the transmission amplitude $T_{m^{\prime}, m}$ from the $m^{\text {th }}$ mode in the entrance lead to the $\left(m^{\prime}\right)^{\text {th }}$ mode in the exit lead. Inside the billiard, i.e., a two-dimensional cavity at constant potential, the trajectories are straight lines which are specularly reflected at hard walls. Despite the conceptual simplicity of the semiclassical description of ballistic transport, recent applications have revealed fundamental difficulties of the semiclassical theory $[4,7-9,14,15,26]$ : among many others, unitarity is badly violated with discrepancies in some cases as large as the conductance fluctuations the theory attempts to describe $[8,9]$. Consequently the correlation $\delta|T|^{2}=-\delta|R|^{2}$ between transmission (or conductance) fluctuations, $\delta|T|^{2}$, and the corresponding fluctuations in the reflection (or resistance), $\delta|R|^{2}$, as a function of the wave number $k$ is broken. Also, the "weak localization" effect is considerably underestimated $[14,15]$.

At first glance, the shortcomings of the semiclassical approximation are not surprising. Hard-walled billiards possess "sharp edges" at the entrance and exit leads. At such points, the length scale $a_{P}$ of spatial variations of the potential approaches zero. Consequently the semiclassical limit $\lambda / a_{P}$ $\ll 1$ cannot be reached no matter how small $\lambda$ (or large $k$ ) is. An obvious improvement of the semiclassical description can be achieved by including into the coupling between the quantum wire and the billiard effects of diffraction at sharp edges within the framework of the Kirchhoff diffraction [6] or Fraunhofer diffraction [7]. However, despite considerable improvement achieved, the fundamental shortcomings are not accounted for. Remarkably, the discrepancies between the semiclassical path-length power spectrum $P_{m^{\prime}, m}^{s c}(\ell)$ and the corresponding quantum path-length spectrum,

$$
P_{m^{\prime}, m}^{q m}(\ell)=\left|\int d k e^{i k \ell} T_{m^{\prime}, m}(k)\right|
$$

are more pronounced for classically regular billiards such as the rectangular [7] or the circular [5,6,26] billiard than for chaotic structures such as the Bunimovich stadium [16$18,26]$. In other words, the path-length spectrum of a regular system where the number of paths grows linearly as a function of length, $N_{c \ell}(\ell) \propto \ell$, is much more sensitive to the approximation of the Feynman path integral by the sum over classical paths than the exponentially proliferating pathlength spectrum, $N_{c \ell}(\ell) \propto \exp (\ell)$, of a chaotic cavity. This observation strongly hints at the lack of missing (non) classical paths as the culprit for the failure. Another hint is provided by the breakdown of the one-to-one correlation between transmission and reflection fluctuations. As classical trajectories that are either ejected through the exit lead contributing to $T$ or return back to the entrance lead contributing to $R$ are disjunct subsets, the inequality $\left(\delta|T|^{2}\right)_{s c} \neq$ $-\left(\delta|R|^{2}\right)_{s c}$ is anything but surprising and indicates that additional paths, pseudopaths referred to in the following, are required to couple these disjunct subsets and thereby restore the correlation between transmission and reflection.

The starting point of our formulation of the semiclassical theory is the close analogy to another class of scattering problems where standard semiclassical theory fails: elastic differential scattering at central potentials displaying pronounced generalized Ramsauer-Townsend interference minima [19]. Following the seminal work of Berry and Mount [20] it could be shown [21] that by including into the semiclassical scattering amplitude, in addition to the classical paths, a small set of pseudopaths an almost perfect agreement with the quantum differential cross section could be achieved. The task is therefore to identify the set of pseudo- 


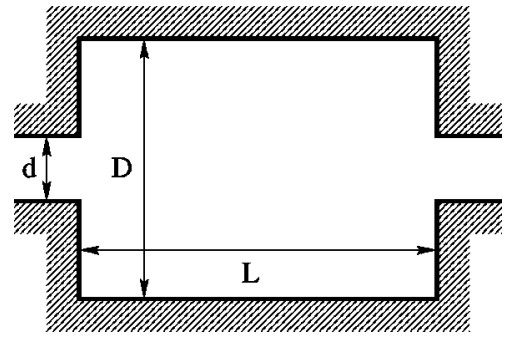

FIG. 1. Rectangular billiard with length $L$, width $D$, and with opposite centered leads of width $d$.

paths for scattering at open billiards. To this end the quantum transmission amplitude is expanded as a multiple scattering series. In its semiclassical limit, propagation between subsequent scattering events can be identified as proceeding along classical paths while each scattering corresponds to a nonclassical diffractive deflection ("kink") at the sharp edges of the lead walls. We perform our analysis for a rectangular billiard with exit and entrance leads at opposite sides of the structure (Fig. 1) for which all paths and pseudopaths can be easily enumerated [34] and the path sum can be performed until convergence is approximately reached. We find excellent agreement with the full quantum calculation for the path-length spectrum and convergence towards the unitarity limit.

The plan of the paper is as follows: In Sec. II we briefly review the standard semiclassical approximation to this problem. In Sec. III we motivate the present multiple scattering approach employed in the enumeration of pseudopaths by revisiting the one-dimensional square well problem. Transcription of this problem to the rectangular billiard allows the enumeration of pseudopaths as discussed in Sec. IV. Explicit expressions for the diffractive amplitudes entering the present semiclassical theory at the level of Fraunhofer diffraction approximation (FDA) are given in Sec. V. Numerical results and comparison with the full quantum results are discussed in Sec. VI followed by a short summary and an outlook to future applications of this approach.

\section{STANDARD SEMICLASSICAL APPROXIMATION}

The conductance $g$ for ballistic transport as a function of the wave number $k$ through an open billiard is given by the Landauer formula [22],

$$
g(k)=\frac{2 e^{2}}{h}\left(\sum_{m=1}^{N} \sum_{m^{\prime}=1}^{N}\left|T_{m^{\prime}, m}(k)\right|^{2}\right),
$$

where $N$ is the number of open modes in the leads (quantum wires) of width $d$. Generically, semiclassical approximations to the transmission amplitudes $T_{m^{\prime} m}$ employ three steps each of which is connected with a stationary phase approximation (SPA) [23]:

(1) The quantum mechanical Feynman propagator $K^{F}\left(\vec{r}^{\prime}, \vec{r}, t\right)[24]$ leads, after application of the SPA, to the semiclassical Van Vleck propagator $K^{V}\left(\vec{r}^{\prime}, \vec{r}, t\right)$, which contains the sum over all classical paths connecting $\vec{r}$ and $\vec{r}^{\prime}$ in time $t[2,25]$.
(2) The Fourier-Laplace transform of $K^{V}\left(\vec{r}^{\prime}, \vec{r}, t\right)$ to the semiclassical Green's propagator $G^{s c}\left(\vec{r}^{\prime}, \vec{r}, E\right)$, which describes the probability amplitude for propagation from $\vec{r}$ to $\vec{r}^{\prime}$ at a fixed energy $E$, is performed by SPA leading to a sum over all classical paths of energy $E$ connecting these two points:

$$
\begin{aligned}
G^{s c}\left(y_{2}, y_{1}, k\right)= & \frac{1}{(2 \pi i)^{1 / 2}} \sum_{y_{1} \rightarrow y_{2}}\left|D_{p}\left(y_{2}, y_{1}, k\right)\right|^{1 / 2} \\
& \times \exp \left[i\left(l_{p}\left(y_{2}, y_{1}\right)-\frac{\pi}{2} \mu_{p}\right] .\right.
\end{aligned}
$$

Here, $l_{p}\left(y_{2}, y_{1}\right)$ denotes the length and $\mu_{p}$ denotes the Maslov index of the path $p$. We denote the transverse coordinates in the entrance/exit lead by $y_{1,2}$ and suppress the corresponding $x$ coordinates in Eq. (2.2). $D_{p}$ denotes the weighting factor (deflection factor) of the path.

(3) The transmission amplitudes (and, equally, reflection amplitudes) from the mode $m$ to the mode $m^{\prime}$ are customarily expressed as the projection of the Green's function [evaluated at the energy $E=\hbar^{2} k^{2} /(2 m)$ ] onto the transverse wave functions $\phi_{m}\left(y_{1}\right)$ and $\phi_{m^{\prime}}\left(y_{2}\right)$ of the incoming and outgoing modes [4],

$$
\begin{aligned}
T_{m^{\prime}, m}(k)= & -\sqrt{v_{x_{2}, m^{\prime}} v_{x_{1}, m}} \int d y_{2} \int d y_{1} \phi_{m^{\prime}}^{*}\left(y_{2}\right) \\
& \times G^{S C}\left(y_{2}, y_{1}, k\right) \phi_{m}\left(y_{1}\right) .
\end{aligned}
$$

This double integral is frequently calculated in the SPA as well. This selects those classical trajectories $p$ that enter the billiard with the quantized angle

$$
\theta_{m}=\sin ^{-1} \frac{m \pi}{d k}
$$

and exit the billiard at the quantized angle

$$
\theta_{m^{\prime}}=\sin ^{-1} \frac{m^{\prime} \pi}{d k}
$$

In an earlier paper [7] we have demonstrated that the SPA in the third step can be avoided by linear expansion of the exponent in Eq. (2.3) allowing for an analytical evaluation of the double integral. Using lead wave functions with longitudinal momentum

$$
k_{m}=\sqrt{k^{2}-|m \pi / d|^{2}}
$$

and transverse wave functions

$$
\Phi_{m}(y)=\sqrt{\frac{2}{d}}\left\{\begin{array}{cc}
\cos \left(\frac{m \pi}{d} y\right), & m \text { odd } \\
\sin \left(\frac{m \pi}{d} y\right) & m \text { even, }
\end{array}\right.
$$

each integral corresponds to a Fraunhofer diffraction integral. This automatically includes diffractive effects on the 
level of the Fraunhofer approximation and yields results comparable to the explicit inclusion of diffractive effects using Kirchhoff theory [6]. The physical picture that emerges is that classical trajectories representing incoming (outgoing) flux no longer enter (exit) the billiard at quantized angles $\theta_{m}$ [see Eq. (2.4)] but with a continuous distribution of angles $\theta$ given by the corresponding diffraction integral. The implementation of this class of diffractive effects for the trajectories leads to a considerable improvement in the transmission and reflection coefficients for low modes and in the pathlength spectrum where the position and height of many peaks could be identified with classical paths. Nevertheless the fundamental difficulties of the semiclassical approximation persist. In particular:

(a) The unitarity condition

$$
\sum_{m^{\prime}=1}^{N}\left|T_{m^{\prime}, m}(k)\right|^{2}+\sum_{m^{\prime}=1}^{N}\left|R_{m^{\prime}, m}(k)\right|^{2}=1
$$

is violated $[7,8,26]$. The fluctuations of the semiclassical conductance $g$ around the exact value remains approximately constant and does not decrease with increasing $k$ (or $\lambda$ $\rightarrow 0$ ).

(b) The path-length power spectrum displays a dramatic overestimate of contributions for long paths $[7-9,26]$ (see Fig. 8 below). The position of the peaks is reproduced remarkably well by the semiclassical approximation. However, the approximately exponential decay of the quantum spectrum contrasts with the inverse linear $\left(l^{-1}\right)$ decay of the semiclassical spectrum.

It is instructive to classify the standard semiclassical theory in terms of the number of the SPA's employed. Depending on the starting point of the description in either time or energy domain one or two SPA's are involved [see Eq. (2.2)] in the propagation. If one neglects diffraction during the injection and emission, two more SPA's are needed. Standard semiclassical approximations (SCA) are therefore characterized by a fixed number of SPA's with the minimum of at least one, that is, when one starts from a time-independent constant energy description and employs a diffraction approximation for the coupling in and out of the billiard structure.

Going beyond the standard approximation requires taking into account nonclassical paths during the propagation inside the billiards in line with the original Feynman propagator. Identifying and enumerating the relevant nonclassical paths to be included can be performed by casting the quantum problem in a multiple scattering problem. The result will be characterized by an increasing number of SPA's with an increasing number of nonclassical paths.

\section{MULTIPLE SCATTERING THEORY: THE ONE-DIMENSIONAL SQUARE WELL POTENTIAL REVISITED}

Our point of departure for the development of the present semiclassical description of quantum transport is the timeindependent quantum scattering wave function for multiple scattering. In order to motivate our strategy for enumerating (a)

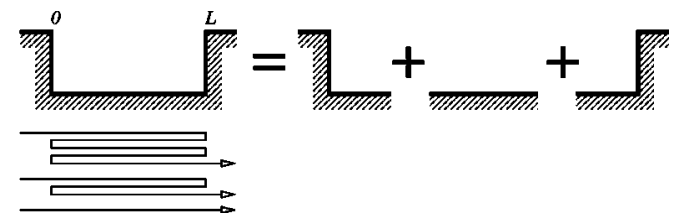

FIG. 2. One-dimensional potential square well and its decomposition into two potential steps (edges) and a region of constant potential.

all classical and nonclassical paths included in the scattering wave function, we revisit the well-known one-dimensional square well problem. Scattering at the square well (SW) is a standard problem treated in most quantum mechanics text books [27] and easily solved by matching the wave function and its derivative at the two edges of a square well potential of width $L$ and depth $-V_{0}$ shown in Fig. 2. The transmission amplitude $T^{(S W)}$ is proportional to the amplitude of the wave function on the right hand side of a square well. In a standard semiclassical approach which is based exclusively on classically allowed paths, there is only one classical path transmitted through the well, and consequently

$$
\left|T_{S C}^{(S W)}\right|=1
$$

at variance with quantum results. This discrepancy is not surprising as at the edges of a well, the semiclassical criterion $\lambda / a_{p} \rightarrow 0$ is violated. We reformulate now the quantum scattering problem in terms of multiple scattering at the two edges. To this end, we consider the square well as a structure composed of three separate substructures, the left edge, the interior of the well, and the right edge (Fig. 2), for each of which we determine separate amplitudes. The transmission amplitude for an incoming wave from the external region from the left with $k^{(e)}=\sqrt{2 E}$ into the interior of the well with $k^{(i)}=\sqrt{2\left(E+V_{0}\right)}$, i.e., forward scattering amplitude at the left edge is given by

$$
t^{(L)}=2 \frac{\sqrt{k^{(i)} k^{(e)}}}{k^{(i)}+k^{(e)}}
$$

Correspondingly, the backscattering (or reflection amplitude) at the left edge from the exterior region back into the exterior region is given by

$$
r^{(L)}=\frac{k^{(e)}-k^{(i)}}{k^{(e)}+k^{(i)}}
$$

The propagation through the interior of the well from the left to the right $(L R)$ [or from the right to the left $(R L)]$ is given by the Green's function

$$
G^{(L R)}\left(x_{R}, x_{L}\right)=e^{i k^{(i)}\left(x_{R}-x_{L}\right)}=e^{i k^{(i)} L}=G^{(R L)}\left(x_{L}, x_{R}\right) .
$$

The corresponding transmission amplitude for the right edge is 


$$
t^{(R)}=2 \frac{\sqrt{k^{(i)} k^{(e)}}}{k^{(e)}+k^{(i)}},
$$

i.e., $t^{(R)}=t^{(L)}=t$. The backscattering amplitude for a wave approaching either edge from the interior is given by

$$
r=\frac{k^{(i)}-k^{(e)}}{k^{(i)}+k^{(e)}},
$$

i.e., $r=-r^{(L)}$. The transmission amplitude through the composite structure, $T$, can now be written as a multiple scattering series of repeated traversals through the structure [see Fig. 2(b)] each calculated with the help of the elementary amplitudes for transmission, reflection, and propagation [Eqs. (3.2)-(3.4)],

$$
\begin{aligned}
T= & t^{(R)}\left[G^{(L R)}(L, 0)+G^{(L R)}(L, 0) r G^{(R L)}(0, L) r G^{(L R)}\right. \\
& \times(L, 0)+\cdots] t^{(L)} \\
= & t^{(R)} G^{(L R)}(L, 0)\left(\sum_{j=0}^{\infty}\left[r G^{(R L)}(0, L) r G^{(L R)}(L, 0)\right]^{j}\right) t^{(L)} .
\end{aligned}
$$

Inserting explicit expressions [Eqs. (3.2)-(3.6)] gives the geometric series

$$
T=t^{2} e^{i k^{(i)} L} \sum_{j=0}^{\infty}\left(r^{2} e^{2 i k^{(i)} L}\right)^{j}
$$

with the result

$$
T=\frac{1}{\cos \left(k^{(i)} L\right)-\frac{i \epsilon}{2} \sin \left(k^{(i)} L\right)},
$$

where

$$
\epsilon=\frac{k^{(e)}}{k^{(i)}}+\frac{k^{(i)}}{k^{(e)}} .
$$

Analogously, the reflection amplitude for the composite structure is given by

$$
\begin{aligned}
R= & r^{(L)}+t^{(L)} G^{(L R)}(L, 0) \sum_{j=0}^{\infty}\left[r G^{(R L)}(0, L) r G^{(L R)}(L, 0)\right]^{j} \\
& \times r G^{(R L)}(0, L) t^{(L)} .
\end{aligned}
$$

Inserting explicit expressions for the substructures yields

$$
\begin{aligned}
R & =-r\left(1-t^{2} e^{2 i L k^{(i)}} \sum_{j=0}^{\infty}\left(r^{2} e^{2 i k^{(i)} L}\right)^{j}\right) \\
& =-r\left(1-\frac{t^{2} e^{2 i k^{(i)} L}}{1-r^{2} e^{2 i k^{(i)} L}}\right) .
\end{aligned}
$$

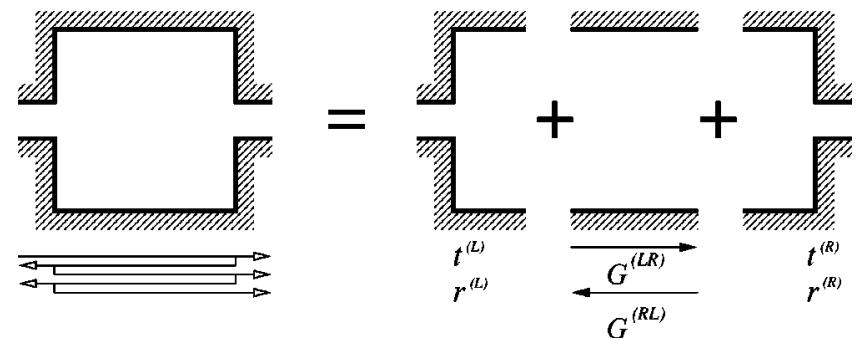

FIG. 3. Decomposition of a rectangular billiard into three separate substructures: a junction from a narrow to a wide constriction, a wide constriction of length $L$, and a junction from a wide to a narrow constriction. Transmission through the junction $=t$, reflection at the junction $=r$, and propagation in between for left to right $=G^{(L R)}$ (or right to left $=G^{(R L)}$ ).

Obviously, these series of multiple scattering at the discontinuous edges converges towards the exact quantum result. Unitarity is trivially satisfied $\left(|R|^{2}+|T|^{2}=1\right)$. Remarkably, we are not aware of a discussion of this intuitive derivation of the square well transmission problem in any standard quantum mechanics textbook. The key point in the present context is now that the formulation of the exact quantum scattering in terms of multiple traversals can be rephrased in terms of a sum over paths, in the following referred to as pseudopaths, which consist of segments of classical paths connected by amplitudes for nonclassical scattering at edges. In one dimension, the semiclassical propagator coincides with the quantum propagator [Eq. (3.4)]. Accordingly, the geometric series [Eqs. (3.8) and (3.12)] can be interpreted as a sum over pseudopaths characterized by an increasing number of traversals through the structure before exiting on either side. Each traversal corresponds to a classical path segment described by a semiclassical propagator. Edge scattering must be described by a quantum scattering amplitude which is an obvious consequence of the fact that at the edge $\lambda / a_{p} \rightarrow \infty$ no matter how large $k$ is and, therefore, the semiclassical limit is never reached. In one dimension, the semiclassical description in terms of a complete set of pseudopaths is naturally equivalent to the full quantum scattering amplitude. The nontrivial generalization of this approach to two (or higher) dimensions is at the core of the present semiclassical approach. In such a case, the sum over pseudopaths is no longer equivalent to the full quantum scattering process but provides a systematic approximation technique to include nonclassical effects (or equivalently, contributions in increasing orders of $\hbar$ ) into the semiclassical description.

\section{TRANSMISSION THROUGH A RECTANGULAR BILLIARD: FROM QUANTUM TO SEMICLASSICAL DESCRIPTION}

The quantum transport problem through a rectangular billiard with opposite leads (Fig. 1) with width $D$ and length $L$ can be formulated in terms of a multiple scattering series in direct analogy to the 1D square well. Accordingly, we decompose the transmission problem into three pieces (Fig. 3): the injection (or transmission) from the entrance lead (or 
quantum wire) to the left into the billiard $t^{(L)}$ the propagation from the left to the right $G^{(L R)}$ or from the right to the left, $G^{(R L)}$, of the cavity, and the emission (or transmission) from the interior into the exit lead to the right $t^{(R)}$. Likewise, the electron approaching the billiard can be reflected at the entrance lead with amplitudes $r^{(L)}$ or can be reflected at each junction from the wide to the narrow constriction if the wave approaches the lead mouth from the interior with amplitude $r$. The scattering amplitudes at each lead mouth representing a discontinuity in the potential become now matrices with indices referring to the transverse mode number, e.g., $t_{n, m}^{(L)}$ where $m$ refers to the mode number in the lead and $n$ to the mode number in the rectangular billiard. These scattering amplitudes at the lead mouth (or junctions between constrictions of different widths) cannot be satisfactorily described by a true semiclassical description at $\lambda / a_{p} \rightarrow \infty$ for any energy of the scattered particle. One can, instead, employ either a full numerical solution of the quantum problem for each junction or, alternatively, an approximate analytic approximation in terms of "diffraction integrals" which are a large $k$ approximation and hence close in spirit to a semiclassical approximation. An explicit evaluation of $t$ and $r$ in terms of Fraunhofer diffraction integrals will be given below.

The multiple scattering expansion of the transmission amplitude $T$ and the reflection amplitude $R$ are given in analogy to Eqs. (3.7) and (3.11) by matrix equations,

$$
T(E)=t^{(R)} G^{(L R)}\left(\sum_{j=0}^{\infty}\left(r G^{(R L)} r G^{(L R)}\right)^{j}\right) t^{(L)},
$$

and

$$
R(E)=r^{(L)}+t^{(L)} G^{(L R)}\left(\sum_{j=0}^{\infty}\left(r G^{(R L)} r G^{(L R)}\right)^{j}\right) r G^{(R L)} t^{(L)} .
$$

Here and in the following we suppress the energy $E$ or wave number $k$ dependence for notational simplicity. For the propagator we choose a mixed representation which is local in $x$, and employs a spectral sum over transverse modes,

$$
G^{(L R)}\left(x_{R}, x_{L}\right)=G^{(R L)}\left(x_{L}, x_{R}\right)=\sum_{n}|n\rangle e^{i k_{n}\left(x_{R}-x_{L}\right)}\langle n| .
$$

The transverse modes in the billiard are described by the wave functions

$$
\phi_{n}(y)=\left\{\begin{array}{l}
\sqrt{\frac{2}{D}} \cos \left(\frac{n \pi}{D} y\right), \quad n \text { odd } \\
\sqrt{\frac{2}{D}} \sin \left(\frac{n \pi}{D} y,\right) \quad n \text { even }
\end{array}\right.
$$

and $x_{R, L}$ are the $x$ coordinates of the right (left) lead with $x_{R}-x_{L}=L$. Equations (4.1) and (4.2) represent a full quantum description of the transport amplitudes $T$ and $R$ in terms of a multiple scattering series. In the following we will investigate its semiclassical limit.

\section{Single and double traversals}

In this subsection we begin to develop an improved semiclassical approximation to the quantum mechanical expressions Eqs. (4.1) and (4.2) for certain classes of short paths, featuring few traversals through the structure. The key feature of this derivation is the transition from discrete mode numbers to continuous angles of incident trajectories.

The amplitude for transmission from mode $m_{L}$ in the left lead to $m_{R}$ in the right lead [Eq. (4.1)] reads explicitly for up to three successive traversals

$$
\begin{aligned}
T_{m_{R}, m_{L}} \approx & \sum_{n} t_{m_{R}, n}^{(R)} e^{i k_{n} L} t_{n, m_{L}}^{(L)}+\sum_{n, n^{\prime}, n^{\prime \prime}} t_{m_{R}, n^{\prime \prime}}^{(R)} e^{i k_{n^{\prime \prime}} L} \\
& \times r_{n^{\prime \prime}, n^{\prime}} e^{i k_{n^{\prime}} L} r_{n^{\prime}, n} e^{i k_{n} L} t_{n, m_{L}}^{(L)}+\cdots
\end{aligned}
$$

with

$$
k_{n}=\sqrt{k^{2}-(n \pi / D)^{2}} .
$$

The crucial step towards a semiclassical approximation to Eqs. (4.1) and (4.5) is now to associate the traversals with classical paths. For clarity we present first the derivation for a path that traverses the cavity just once corresponding to the first term in Eq. (4.5). We then extend our derivation to multiple traversals through the cavity and show that the sum over multiple traversals can be performed by mapping the problem on combinatorics of lattice vectors in an extended zone scheme of a rectangular lattice. The method of the extended zone scheme has also been used to determine a semiclassical expansion of the transmission amplitudes in terms of a finite number of continued fractions [28].

In order to associate the first term in Eq. (4.5) with classical paths we have to map the transverse quantum number $n$ onto an injection angle $\theta$. For this task we employ the Poisson sum formula [29]. Without loss of generality we take here and in the following $m_{L}$ and $m_{R}$ to be odd integers. Because of inversion symmetry, the subspaces of even and odd mode number completely decouple. Analogous expressions can be derived for even $m_{L, R}$. The single traversal ( $\left.\mathrm{t} 1\right)$ contribution becomes

$$
\begin{aligned}
T_{m_{R}, m_{L}}^{(t 1)}(k)= & \frac{1}{4} \sum_{\alpha=-\infty}^{\infty} \int_{-\infty}^{\infty} t_{m_{R}}(\nu) t_{m_{L}}(\nu) \\
& \times e^{i L \sqrt{k^{2}-\left(\frac{\nu \pi}{D}\right)^{2}}+i \alpha \pi(\nu-1)} d \nu .
\end{aligned}
$$

The sum over integers $n$ can be replaced by an integral over the continuous variable $\nu$. Since the (discrete) mode numbers $n$ are associated with quantized angles $\sin \theta=n \pi /(k D)$, the integration over the variable $\nu$ can be associated with an angle integration via the substitution

$$
\nu=\frac{k D}{\pi} \sin (\theta) \rightarrow d \nu=\frac{k D}{\pi} \cos (\theta) d \theta .
$$

If we restrict $\theta$ to real angles, the variable $\nu$ is restricted to the interval $[-k D / \pi, k D / \pi]$. Since values outside this interval correspond to evanescent rather than propagating 
waves, we neglect the latter which would make only an exponentially small contribution to the transport for large $L$. With the substitution [Eq. (4.7)] we arrive at

$$
\begin{aligned}
T_{m_{R}, m_{L}}^{(1)}= & \frac{k D}{4 \pi} \sum_{\alpha=-\infty}^{\infty} \int_{-\pi / 2}^{\pi / 2} t_{m_{R}}^{d}(\theta) t_{m_{L}}^{d}(\theta) \\
& \times e^{i k[L \cos (\theta)+\alpha D \sin (\theta)]-i \pi \alpha} \cos (\theta) d \theta,
\end{aligned}
$$

where $t_{m_{L}}^{d}(\theta), t_{m_{R}}^{d}(\theta)$ are the injection (ejection) amplitudes and the transverse mode index $n$ is converted to a continuous angle variable $\theta$. For these amplitudes we will later employ the FDA in line with previous semiclassical treatments [7]. The action in the exponent of Eq. (4.8)

$$
S(\theta)=k[L \cos (\theta)+\alpha D \sin (\theta)]
$$

has a simple geometric interpretation in the extended zone scheme of the rectangular lattice [Fig. 4(a)]. The action is that of a path connecting the center of the left side of the unit cell, the position of the entrance lead, with the center of the right side of the $\alpha^{\text {th }}$ replica of the unit cell in the transverse direction. The path possesses at most one discontinuity, i.e., a discontinuous displacement of the trajectory $\Delta \vec{r}$ perpendicular to the direction of propagation such that $\vec{k} \Delta \vec{r}=0$ and no contribution to the classical action is accumulated along the displacement $\Delta \vec{r}$. Note that Eq. (4.8) is, apart from neglecting evanescent modes, equivalent to the original quantum expression for single traversals. This set of straight-line paths connecting the entrance lead with any one of the replicas of the exit lead featuring no more than one lateral displacement amounts to the representation of a full path sum of the Feynman propagator for this process.

We derive now the semiclassical limit of Eq. (4.8) by applying the SPA. The SPA is valid if the action $S(\theta)$ is rapidly varying on a scale of $2 \pi$, i.e., if $k L \gg \pi$ or $k D \gg \pi$. Since for a given mode $n, k \simeq n \pi / d$, these conditions are only fulfilled for all $n$ provided that $D / d \gg 1$ or $L / d \gg 1$, i.e., if at least one of the linear dimensions of the rectangular billiard is large compared to the lead width. With this restriction in mind, we obtain from the stationary phase condition

$$
0=S^{\prime}(\theta)=k[-L \sin (\theta)+\alpha D \cos (\theta)]
$$

the "stationary" angles

$$
\theta_{\alpha}=\arctan \frac{\alpha D}{L}
$$

which coincide with the angles of the classical straight-line paths of length $l_{\alpha}$ connecting the lattice point of the entrance lead with the lattice point of the $\alpha^{t h}$ replica of the exit lead [Fig. 4(b)]. The stationarity condition eliminates the discontinuous displacement and renders the paths fully classical. The number $\alpha$ corresponds to the number of horizontal zone boundaries the trajectory has crossed. The second derivative yields the deflection factor for each path (a)

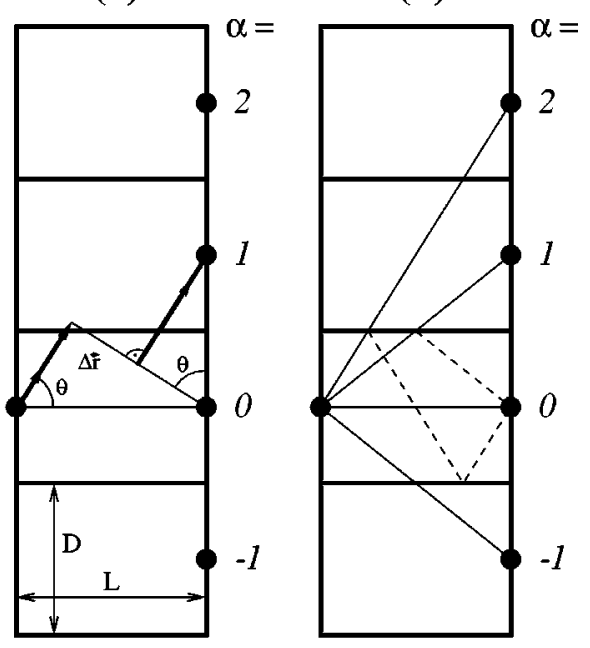

(c)

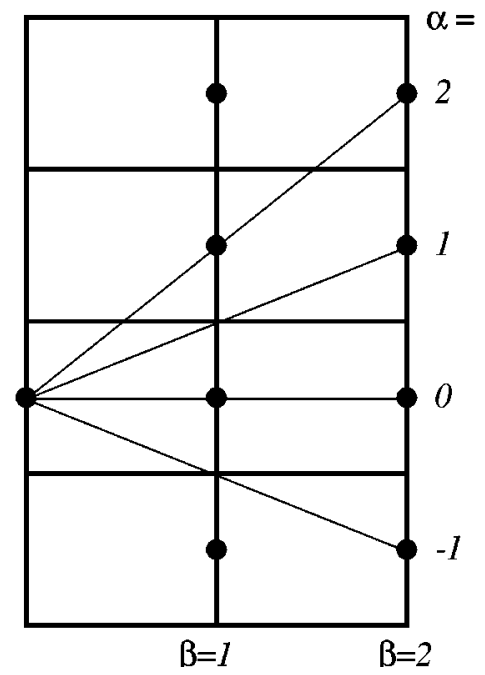

FIG. 4. Examples of classical and nonclassical paths for single and double traversals depicted in the extended zone scheme (a) nonclassical path for single traversal with one lateral displacement representing the full Feynman path sum. (b) Classical paths for single traversal after stationary phase approximation (dashed line: classical paths in the fundamental unit cell). (c) Classical paths for double traversal contributing to reflection.

$$
\begin{aligned}
S^{\prime \prime}(\theta) & =-k[L \cos (\theta)+\alpha D \sin (\theta)] \\
& =-\frac{k}{L}\left(L^{2}+\alpha^{2} D^{2}\right) \cos \left(\theta_{\alpha}\right)=-k l_{\alpha} .
\end{aligned}
$$

We thus obtain the semiclassical transmission coefficient for single traversals

$$
\begin{aligned}
T_{m_{R}, m_{L}}^{(t 1)}= & \frac{k D}{2} \sqrt{\frac{1}{2 \pi i_{\alpha}}} \sum_{\alpha=-\infty}^{\infty} \sqrt{\frac{1}{k l_{\alpha}}} e^{i\left(k l_{\alpha}-\pi \alpha\right)} t_{m_{R}}^{d}\left(\theta_{\alpha}\right) \\
& \times \cos \theta_{\alpha} t_{m_{L}}^{d}\left(\theta_{\alpha}\right)
\end{aligned}
$$

The phase $\pi \alpha$ in Eq. (4.13) is associated with the Maslov index for $\alpha$ crossings of the "hard-wall" boundaries (i.e., 
horizontal zone boundaries) the trajectory has crossed on its way to the $\alpha^{t h}$ exit lattice point [see Fig. 4(b)]. The classical path sum in Eq. (4.13) corresponds to a sum over all lattice vectors connecting the lattice point of the entrance lead with exit points in the $\alpha^{\text {th }}$ unit cell.

The next term in the multiple scattering expansion [Eqs. (4.1), (4.2)] corresponds to the double traversal $t 2$. A $t 2$ contribution does not appear in the expansion of the transmission amplitude [Eq. (4.5)]. Note that odd (even) number of traversal correspond to transmission (reflection). Accordingly, the lowest-order reflection amplitude is

$$
R_{m_{L^{\prime}, m_{L}}^{(t 2)}}=\sum_{n, n^{\prime}} t_{m_{L^{\prime}, n^{\prime}}^{(L)}}^{\left(i k_{n^{\prime}} L\right.} r_{n^{\prime}, n} e^{i k_{n} L} t_{n, m_{L}}^{(L)}
$$

Before again applying the Poisson formula, we decompose the internal reflection amplitude at the vertical wall into the geometric hard-wall reflection amplitude of the closed billiard and the diffractive amplitude for the lead opening

$$
r_{n^{\prime}, n}=-\delta_{n^{\prime}, n}+r_{n^{\prime}, n}^{d}
$$

The term $-\delta_{n^{\prime}, n}$ corresponds to geometric scattering at the hard vertical wall of the closed billiard including its phase jump (Maslov index) while the amplitude $r^{d}$ corresponds to the diffractive scattering at the mouth of the lead. Accordingly, Eq. (4.14) can be decomposed into terms involving different numbers of diffractive scatterings inside the cavity,

$$
\begin{aligned}
R_{m_{L^{\prime}, m_{L}}}^{(t 2)}= & \sum_{n} t_{m_{L^{\prime}, n}}^{(L)} e^{i\left(2 k_{n} L-\pi\right)} t_{n, m_{L}}^{(L)} \\
& +\sum_{n, n^{\prime}} t_{m_{L^{\prime}, n^{\prime}}}^{(L)} e^{i k_{n^{\prime}} L} r_{n^{\prime}, n}^{d}(k) e^{i k_{n} L} t_{n, m_{L}}^{(L)}
\end{aligned}
$$

The first term with zero internal diffractive scattering is formally completely equivalent to the first term for the transmission amplitude in Eq. (4.5), while the second term with one internal diffractive scattering contains one additional sum over transverse mode numbers. The semiclassical approximation for the zero-diffraction term proceeds as above and yields in analogy to Eq. (4.13)

$$
\begin{aligned}
R_{m_{L^{\prime}}, m_{L}}^{(t 2)} & (\text { zero diffraction) } \\
= & \frac{k D}{2} \sqrt{\frac{1}{2 \pi i}} \sum_{\alpha=-\infty}^{\infty} \sqrt{\frac{1}{k l_{\alpha, \beta}}} \\
& \times e^{i\left[k l_{\alpha, \beta}-\pi \alpha-(\beta-1) \pi\right]} t_{m_{L^{\prime}}{ }^{\prime}}\left(\theta_{\alpha}, k\right) \cos \theta_{\alpha} t_{m_{L}}^{d}\left(\theta_{\alpha}, k\right),
\end{aligned}
$$

with $l_{\alpha, \beta}=\sqrt{(\beta L)^{2}+(\alpha D)^{2}}$ and $\beta=2$. The index $\beta$ in Eq. (4.17) corresponds in the extended zone scheme to the lattice coordinate in the horizontal direction [Fig. 4(c)]. The zerodiffraction paths correspond to straight-line rays emanating from the entrance point $(\alpha=0, \beta=0)$ reaching the lattice

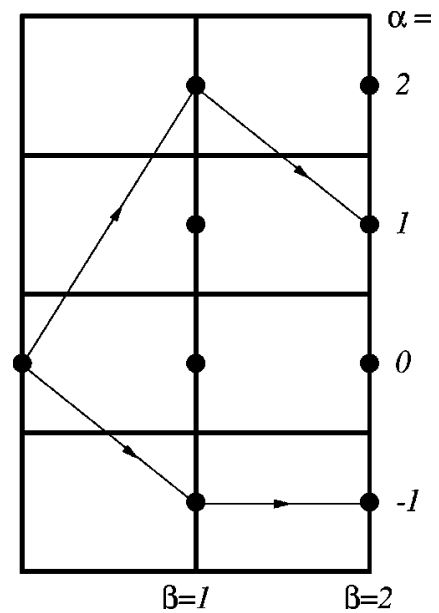

FIG. 5. Two examples for pseudopaths consisting of classical path segments and "kinks," i.e., diffractive scatterings at lattice coordinates representing the lead mouth.

points $(\alpha, \beta=2)$. More generally, $\beta=$ even correspond to reflected paths, while $\beta=$ odd correspond to transmitted paths.

The one-diffraction contribution in Eq. (4.16) can now be converted into contributions from pseudopaths, more precisely, of two segments of classical paths by applying the Poisson formula twice to eliminate the summation over $n$ and $n^{\prime}$. Each Poisson sum, in the semiclassical limit, requires an additional SPA. A straightforward evaluation leads to

$$
\begin{aligned}
R_{m_{L}{ }^{\prime}, m_{L}}^{(t 2)} & (\text { one diffraction) } \\
= & \left(\frac{k D}{2}\right)^{2}\left(\sqrt{\frac{1}{2 \pi i}}\right)^{2} \sum_{\alpha_{2}=-\infty}^{\infty} \sum_{\alpha_{1}=-\infty}^{\infty} \sqrt{\frac{1}{k l_{\alpha_{2}}}} \sqrt{\frac{1}{k l_{\alpha_{1}}}} \\
& \times e^{i\left[k\left(l_{\alpha_{1}}+l_{\alpha_{2}}\right)-\pi\left(\alpha_{1}+\alpha_{2}\right)\right]} t_{m_{L}{ }^{\prime}}^{d}\left(\theta_{\alpha_{2}}, k\right) \\
& \times \cos \theta_{\alpha_{2}} r^{d}\left(\theta_{\alpha_{2}}, \theta_{\alpha_{1}}, k\right) \cos \theta_{\alpha_{1}} t_{m_{L}}^{d}\left(\theta_{\alpha_{1}}, k\right)
\end{aligned}
$$

where

$$
\theta_{\alpha_{i}}=\arctan ^{-1} \frac{\alpha_{i} D}{L}
$$

are the continuous angle variables replacing the mode numbers $n_{i}$ in $r^{d}$. The path described by Eq. (4.18) corresponds to two classical path segments (Fig. 5), one connecting the origin $(0,0)$ with the lattice point $\left(\alpha_{1}, \beta=1\right)$ followed by one that connects $\left(\alpha_{1}, \beta=1\right)$ with $\left(\alpha_{2}, \beta=2\right)$. The two segments are joined through a diffractive scattering amplitude $r^{d}$ which changes directions of the path, thereby introducing nonclassical "kinks" into an otherwise classical path. For convenience we absorb prefactors in Eqs. (4.13) and (4.18) by rescaling the diffractive scattering amplitude $r^{d}$ and the injection and emission amplitudes $t^{d}$ as

$$
t_{m}(\theta)=\sqrt{k D \cos \theta / 2} t_{m}^{d}(\theta),
$$




$$
r\left(\theta^{\prime}, \theta\right)=\sqrt{\cos \theta^{\prime} \cos \theta} \frac{k D}{2} r^{d}\left(\theta^{\prime}, \theta\right)
$$

Explicit expressions will be given in the following section within the framework of the Fraunhofer diffraction approximation (FDA). It will be shown that $t_{m}^{d}(\theta)$ contains an inverse factor of $\sqrt{D}$ and $r^{d}\left(\theta^{\prime}, \theta\right)$ contains an inverse factor of $D$. After rescaling through Eqs. (4.20a) and (4.20b) this leads to an independence of the scattering amplitudes on the cavity dimension $D$. This important feature is, however, not restricted to the FDA, but can be proven generally for diffraction amplitudes in the far-field region. Of course, the far-field approximation is only valid if the two leads are sufficiently far apart. This requires that $k D \gg \pi$ or $k L \gg \pi$, a condition that was already encountered in the derivation of Eq. (4.10).

After rescaling, the zero-order diffraction [or zero-kink contribution, Eq. (4.17)] transforms to

$$
\begin{aligned}
R_{m_{L^{\prime}, m_{L}}}^{(2 t)} \text { (zero diffraction) } & \left(\frac{1}{2 \pi i}\right)^{1 / 2} \sum_{\alpha=-\infty}^{\infty} \sqrt{\frac{1}{k \ell_{\alpha, \beta=2}}} e^{i\left[k \ell_{\alpha, 2}-\pi(\alpha+1)\right]} \\
& \times t_{m_{L^{\prime}}}\left(\theta_{\alpha}\right) t_{m_{L}}\left(\theta_{\alpha}\right)
\end{aligned}
$$

The one-kink contribution becomes

$$
\begin{aligned}
& R_{m_{L^{\prime} m_{L}}}^{(2 t)} \text { (one diffraction) } \\
& \quad=\left(\frac{1}{2 \pi i}\right)^{1 / 2}\left(\frac{1}{2 \pi i}\right)^{1 / 2} \sum_{\alpha_{1}=-\infty}^{\infty} \sum_{\alpha_{2}=-\infty}^{\infty} \sqrt{\frac{1}{k \ell_{\alpha_{1}, 0}}} \sqrt{\frac{1}{k \ell_{\alpha_{2}, 0}}} e^{i\left[k\left(\ell_{\alpha_{1}}+\ell_{\alpha_{2}}\right)-\pi\left(\alpha_{1}+\alpha_{2}\right)\right]} t_{m_{L}{ }^{\prime}}\left(\theta_{\alpha_{2}}\right) r\left(\theta_{\alpha_{2}}, \theta_{\alpha_{1}}\right) t_{m_{L}}\left(\theta_{\alpha_{1}}\right) .
\end{aligned}
$$

With Eqs. (4.18), (4.21), and (4.22) we have all ingredients at our disposal to write down the complete multiple scattering series.

\section{Generalization to arbitrary paths}

Complete multiple scattering series [Eqs. (4.1) and (4.2)] can be formulated in the semiclassical limit as a sum over lattice vectors connected by an increasing number, $K$, of kinks. We first note that all amplitudes with a different number of traversals [Eqs. (4.13), (4.21)] and different numbers of kinks [Eq. (4.22)] contain as an ingredient the 2D semiclassical Green's function [Eq. (2.1)] for propagation along a lattice vector $(\Delta \alpha, \Delta \beta)$ of the rectangular lattice,

$$
G^{S C}(\Delta \alpha, \Delta \beta)=\left(\frac{1}{2 \pi i k \ell_{\Delta \alpha, \Delta \beta}}\right)^{1 / 2} e^{i\left[k \ell_{\Delta \alpha, \Delta \beta}-\pi(\Delta \alpha+\Delta \beta-1)\right]} .
$$

For an arbitrary $S$ matrix element (which stands for either a reflection amplitude $R_{m_{L}{ }^{\prime}, m_{L}}$ or a transmission amplitude $T_{m_{R}, m_{L}}$ ) we have

$$
S_{m^{\prime}, m}=S_{m^{\prime}, m}^{(0)}+\sum_{K>0}^{\infty} S_{m^{\prime}, m}^{(K)}
$$

with a zero-kink $(K=0) \mathrm{S}$ matrix element

$$
S_{m^{\prime}, m}^{(0)}=\sum_{\alpha=-\infty}^{\infty} \sum_{\beta \geqslant 1} t_{m^{\prime}}\left[\theta(\alpha, \beta) G^{S C}(\alpha, \beta) t_{m}[\theta(\alpha, \beta)],\right.
$$

where $\theta(\alpha, \beta)$ is given by Eq. (4.28d). The sum extends over all lattice points $(\alpha, \beta)$ of the right half plane with the restriction that $\beta$ is odd if the matrix element $S_{m^{\prime}, m}$ stands for a transmission amplitude and $\beta$ is even if it represents a reflection amplitude. Equation (4.25) is equivalent to the standard semiclassical approximation, i.e.,

$$
S_{m^{\prime}, m}^{(0)} \simeq S_{m^{\prime}, m}^{S C A}
$$

when diffractive injection and emission [7] is included. However, one important difference is worth noting: The splitting of the reflection amplitude for vertical walls into a geometric and a diffractive term [Eq. (4.15)] corresponds to the treatment of the lead opening as a pointlike scatterer. Therefore, $S_{m^{\prime}, m}^{(0)}$ does not include the effect of geometric path shadowing, i.e., the elimination of longer paths due to their premature exit through the opening of finite width. This leads to contributions of paths to $S_{m^{\prime}, m}^{(0)}$ which are not present in $S_{m^{\prime}, m}^{S C A}$. However, the amplitudes of these paths are reduced by interference with higher-order diffractive paths of the same or very similar length.

Geometric reflections at the open lead are closely related to the so-called "ghost paths" which were introduced by Schwieters et al. [6], in order to treat diffractive backscattering at the open lead mouths using Kirchhoff diffraction theory. However, in [6] only specularly reflected ghost-paths corresponding to straight lines in the extended zone scheme were used. They comprise only a small subset of the complete set of kink paths (nonspecularly reflected pseudopaths). It will become evident below that the whole set of kink-paths is needed in order to describe properly the path-length power spectrum and to restore unitarity.

With an increasing number of kinks, Eq. (4.24) represents corrections to the standard semiclassical approximation of increasing order $(k D)^{-K / 2}$, or equivalently $\hbar^{K / 2}$. For a given $K$, the $K$-kink scattering amplitude is given by 


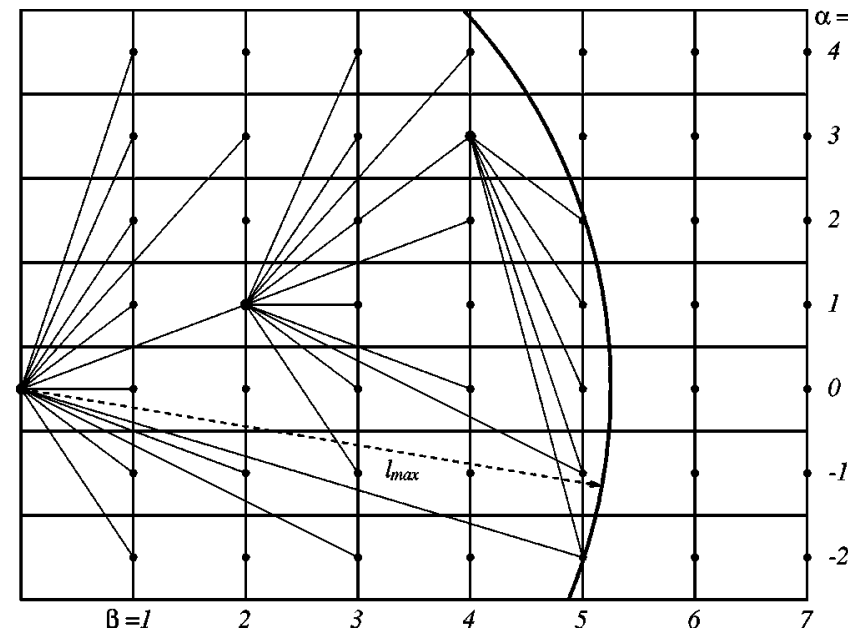

FIG. 6. Proliferation of pseudopaths. The semicircle with radius $\ell_{\text {max }}$ denotes the maximum path length included.

$$
\begin{aligned}
S_{m^{\prime}, m}^{(K)}= & \sum_{\Delta \alpha_{1}=-\infty}^{\infty} \sum_{\Delta \beta_{1} \geqslant 1}^{\infty} \ldots \sum_{\Delta \alpha_{K+1}=-\infty}^{\infty} \sum_{\Delta \beta_{K+1} \geqslant 1}^{\infty} t_{m^{\prime}} \\
& \times\left[\theta\left(\Delta \alpha_{1}, \Delta \beta_{1}\right)\right] \prod_{K^{\prime}=1}^{K}\left\{G^{S C}\left(\Delta \alpha_{K^{\prime}}, \Delta \beta_{K^{\prime}}\right)\right. \\
& \left.\times r\left[\theta\left(\Delta \alpha_{K^{\prime}+1}, \Delta \beta_{K^{\prime}+1}\right), \theta\left(\Delta \alpha_{K^{\prime}}, \Delta \beta_{K^{\prime}}\right)\right]\right\} \\
& \times G^{S C}\left(\Delta \alpha_{K+1}, \Delta \beta_{K+1}\right) t_{m}\left[\theta\left(\Delta \alpha_{K+1}, \Delta \beta_{K+1}\right)\right] .
\end{aligned}
$$

We have introduced the following abbreviations

$$
\Delta \alpha_{i}=\alpha_{i}-\alpha_{i-1}
$$

with $\alpha_{0}=0$ and $\Sigma \Delta \alpha_{i}=\alpha$,

$$
\Delta \beta_{i}=\beta_{i}-\beta_{i-1} \geqslant 0
$$

with $\beta_{0}$ and $\Sigma_{i} \Delta \beta_{i}=\beta$,

$$
\begin{gathered}
\ell_{\Delta \alpha, \Delta \beta}=\sqrt{(\Delta \beta L)^{2}+(\Delta \alpha D)^{2}}, \\
\theta(\Delta \alpha, \Delta \beta)=\tan ^{-1}\left(\frac{\Delta \alpha D}{\Delta \beta L}\right) .
\end{gathered}
$$

The geometric picture underlying Eq. (4.27) is the sum over all pseudopaths consisting of all classical paths of arbitrary length joined by $K$ kinks. In other words: Each lattice point reached by the ray of classical trajectories emanating from the entrance lead into the positive half plane spawns a new bundle of classical trajectories into the positive half plane with positive $\Delta \beta$. This process is repeated $K$ times (Fig. 6). Apart from the diffractive amplitudes $r$ and $t$, Eq. (4.27) contains only quantities calculated from classical dynamics. We refer to this semiclassical approximations in the following as pseudopath semiclassical approximation (PSCA). The present formulation therefore, extends the semiclassical ap- proximation by including higher-order contributions in $\hbar$. It is important to point out some of the limitations of validity of the present formulation:

(a) While the present approach allows the systematic inclusion of certain classes of corrections of increasing order in $(k \ell)^{-K / 2}$ and thus of increasing order in the effective $\hbar^{K / 2}$, this expansion does not assure the inclusion of all corrections to a given order. This is due to the fact that an independent approximation of the diffractive amplitudes $r$ and $t$ is required which is not yet specified. The latter may allow for another expansion in $(k d)^{-n}$ which would, in turn, provide additional contribution to a given order in $\hbar$. Moreover, in general, cross terms of the form $(k d)^{-n}(k \ell)^{-m}$ may appear.

(b) The present analysis assumes that the leads are the only interior points where diffractive scattering occurs. This is correct not only for the rectangular billiard explicitly treated here but also a large class of other structures such as the circle or the Bunimovich stadium. However, for other structures containing diffractive edges (interior angles $\geqslant \pi$ ) or concave curvatures additional classes of pseudopathes must be included.

(c) On a more technical level, the present definition assumes that the SPA evaluations [e.g., Eq. (4.8)] can be performed under the assumption that the diffractive amplitudes $t$ and $r$ are slowly varying functions. Depending on the chosen approximation for the latter and on the size of $(k d)$, this may lead to additional corrections in the SPA integrals and in Eqs. (4.24)-(4.27). Clearly, the latter restriction can be removed once the analytic approximation to $r$ and $t$ is specified.

In the path sum Eq. (4.27) organized in terms of the number of kinks the total path length

$$
\ell_{p}=\sum_{i} \ell_{\Delta \alpha_{i}, \Delta \beta_{i}}
$$

and hence, the classical action $S_{p}=k \ell_{p}$ is not fixed. In a numerical implementation of the semiclassical theory, it is advantageous to include only terms up to a given maximum action $S_{p} \leqslant S_{p}^{\max }$, or equivalently

$$
\ell_{p} \leqslant \ell_{\max }
$$

The pseudo-path sum Eq. (4.24) can be reformulated in terms of the fixed maximum total length and variable $K$,

$$
S_{m^{\prime}, m}\left(\ell_{p} \leqslant \ell_{\text {max }}\right)=\sum_{K=0}^{K_{\max }} S_{m^{\prime}, m}^{(K)}\left(\ell_{p} \leqslant \ell_{\text {max }}\right) .
$$

With the restriction (4.30) also the number of included kinks is restricted to $K \leqslant K_{\max }$. Furthermore, the sum over all lattice vectors $\Delta \alpha_{i}, \Delta \beta_{i}$ appearing in Eqs. (4.25) and (4.27) is restricted as well. Geometrically, Eq. (4.31) can be easily visualized (Fig. 6). The length of all rays of classical paths emanating from the entrance lead into the positive half plane is limited to $\ell_{p} \leqslant \ell_{\text {max }}$. At all lattice points inside the semicircle of radius $\ell_{\max }$ each classical trajectory spawns a new generation of rays of trajectories into a new semicircle of radius $\ell_{\max }$ centered about a respective lattice point. However, only the subset of those is included in the sum [Eq. 
(4.31)] for which the target lattice point lies again in the original semicircle [i.e., which is subject to the constraint (4.29)]. This process can be repeated up to a maximum number given by the maximum number of lateral displacements

$$
\beta_{\max }=\left[\frac{\ell_{\max }}{L}\right],
$$

where the bracket stands for the largest integer less than the argument with the additional constraint that $\beta_{\max }$ is odd (even) for transmission (reflection). Equation (4.32) follows from the fact that for each daughter generation of spawned trajectories $\Delta \beta \geqslant 1$. Consequently, $K_{\max } \leqslant \beta_{\max }-1$.

The important point to be noted is that the number of pseudopaths included in Eq. (4.31) proliferates exponentially with $\ell_{\max }$ while the number of classical paths, i.e., the number of paths included in $S_{m^{\prime}, m}^{(0)}$ grows only linearly. The loss of information on an exponentially growing number of diffractive pseudopaths is at the core of the failure of the standard semiclassical approximation, in particular for the pathlength power spectrum and the unitarity.

\section{FRAUNHOFER DIFFRACTION APPROXIMATION}

The expression for the scattering matrix in terms of the semiclassical sum of pseudopaths contains amplitudes $t$ and $r$ for injection, emission, and diffractive scattering at the lead mouth. Because of the sharp edges with $\lambda / a_{p} \rightarrow \infty$ for all $k$, calculation of $r$ and $t$ is not feasible within standard semiclassical theory. An applicable approximation close in spirit to a semiclassical approximation is a diffraction approximation valid for short wavelength, i.e., $k d \gg 1$ [30]. We use in the following the Fraunhofer diffraction approximation which we have previously used within the framework of the standard semiclassical approximation. Accordingly, both $t^{d}$ and $r^{d}$ can be expressed in terms of the fundamental diffraction integral (for odd $n$ ),

$$
I_{i}^{F D A}(\theta, n)=\frac{1}{\sqrt{2 w_{i}}} \int_{-d / 2}^{d / 2} e^{i\left(k_{n}^{i}+k \sin \theta\right) y} d y
$$

with

$$
\begin{gathered}
w_{1}=d, \\
w_{2}=D, \\
k_{n}^{1}=n \pi / d, \\
k_{n}^{2}=n \pi / D .
\end{gathered}
$$

The index $i=1$ refers to the wave incident from the quantum wire while the index $i=2$ refers to the wave approaching the mouth of the lead from the inside. Evaluation of the integral for odd integer yields

$$
I_{i}^{F D A}(\theta, n)=\sqrt{\frac{2}{w_{i}}}\left(\frac{\sin \left[\left(k_{n}^{i}+k \sin \theta\right) d / 2\right]}{k_{n}^{i}+k \sin \theta}\right) .
$$

The amplitude for injection (emission) can be expressed for quantized angles in terms of Eq. (5.3) as

$$
\begin{aligned}
t_{n, m}^{F D A}= & \sqrt{\frac{2}{D}}\left\{I_{1}^{F D A}\left[\sin ^{-1}\left(\frac{\pi n}{k D}\right), m\right]\right. \\
& \left.+I_{1}^{F D A}\left[\sin ^{-1}\left(\frac{\pi n}{k D}\right),-m\right]\right\} .
\end{aligned}
$$

For continuous angles the amplitude for injection or emission appearing in Eq. (4.8) is accordingly given by

$$
t_{m}^{d}(\theta)=t_{m}^{F D A}(\theta)=\sqrt{\frac{2}{D}}\left[I_{1}^{F D A}(\theta, m)+I_{1}^{F D A}(\theta,-m)\right] .
$$

After rescaling according to Eq. (4.20) the injection or emission amplitude appearing in the PSCA [Eq. (4.27)] becomes

$$
\begin{aligned}
t_{m}(\theta)= & \sqrt{k D \cos \theta / 2} t_{m}^{d}(\theta) \\
= & \sqrt{\frac{2 \cos \theta}{k d}}\left[\frac{\sin \left[\left(k_{m}^{(1)}+k \sin \theta\right) d / 2\right]}{\sin \theta+k_{m}^{(1)} / k}\right. \\
& \left.+\frac{\sin \left[\left(k \sin \theta-k_{m}^{(1)}\right) d / 2\right]}{\sin \theta-k_{m}^{(1)} / k}\right] .
\end{aligned}
$$

The diffractive part of the reflection amplitude [Eq. (4.15)] back into the billiard is given in Fraunhofer approximation by

$$
\begin{aligned}
r_{n^{\prime}, n}^{d}=r_{n^{\prime}, n}^{F D A}= & \sqrt{\frac{2}{D}}\left\{I_{2}^{F D A}\left[\theta^{\prime}=\sin ^{-1}\left(\frac{\pi n^{\prime}}{k D}\right), n\right]\right. \\
& \left.+I_{2}^{F D A}\left(\theta^{\prime}, n\right)\right\} .
\end{aligned}
$$

In the semiclassical limit discussed in the previous section, Eq. (5.7) simplifies further. When the transformation from the discrete quantum numbers $\left(n^{\prime}, n\right)$ to continuous angle variables $\left(\theta^{\prime}, \theta\right)$ via the Poisson formula is employed, $k_{n}^{\prime}$ is mapped onto the continuous function $k \sin \theta^{\prime}$. If we extend the range of angles to $\left(-\pi / 2 \leqslant \theta^{\prime} \leqslant \pi / 2\right)$, the two terms in Eq. (5.7) become equivalent and reduce to

$$
r^{d}\left(\theta^{\prime}, \theta\right)=2 \sqrt{\frac{2}{D}} I_{2}^{F D A}\left(\theta^{\prime}, \theta\right)=\frac{4}{D} \frac{\sin \left[k d / 2\left(\sin \theta^{\prime}+\sin \theta\right)\right]}{k\left(\sin \theta^{\prime}+\sin \theta\right)} .
$$

After rescaling [Eq. (4.20)], the diffraction or kink amplitude entering the PSCA [Eq. (4.27)] is given by

$$
\begin{aligned}
r\left(\theta^{\prime}, \theta\right) & =\frac{k D}{2} \sqrt{\cos \theta^{\prime} \cos \theta} r^{d}\left(\theta^{\prime}, \theta\right) \\
& =2 \sqrt{\cos \theta^{\prime} \cos \theta} \frac{\sin \left[k d / 2\left(\sin \theta^{\prime}+\sin \theta\right)\right]}{\sin \theta^{\prime}+\sin \theta} .
\end{aligned}
$$


The amplitudes for injection or emission $t_{m}(\theta)$ [Eq. (5.6)] and for internal diffractive reflection $r\left(\theta^{\prime}, \theta\right)$ [Eq. (5.9)] entering our semiclassical theory possess the remarkable feature that they are independent of the geometric parameter of the cavity (i.e., $D$ ). This observation indicates that the semiclassical multiple scattering expansion [Eqs. (4.24) and (4.27)] should be applicable irrespective of the geometry of the cavity.

\section{NUMERICAL RESULTS}

We present in the following numerical results within the framework of the present semiclassical theory. We focus on two properties for which previous semiclassical descriptions faced major problems: unitarity and the path-length power spectrum. We compare our results with the previously proposed semiclassical description in which both injection and emission was treated in FDA, but only classical paths were included for propagation inside the rectangular billiard. This corresponds to the approximation $S_{m^{\prime}, m} \approx S_{m^{\prime}, m}^{(0)}$ [Eq. (4.24)] with $t_{m}(\theta)$ given by Eq. (5.6). We also perform detailed comparison with quantum calculations. For the latter we exploit the correspondence between the semiclassical and the quantum multiple scattering series. Since any semiclassical calculation can only be performed for up to a certain maximum path length $\ell_{\max }$ corresponding to a maximum lateral lattice displacement $\beta_{\max }$ [Eq. (4.32)], errors due to the truncation of the contributing paths should be disentangled from the errors due to the semiclassical approximation itself. This is easily accomplished within the present multiple scattering theory as the corresponding quantum multiple scattering expansion [Eqs. (4.1) and (4.2)] can be truncated at the same $\beta_{\max }$ so that semiclassical and quantum calculations can be directly compared at the same level of truncation. Moreover, the quantum multiple scattering series depends also on the same substructure amplitudes $t$ and $r$ for injection, emission, and internal reflections. Calculating the latter quantum mechanically and truncating the multiple scattering series at $\beta_{\max }=11$ yields the result for the unitarity (Fig. 7) labeled as TQM. This truncated quantum result is only approximately unitary $(\approx 0.95)$ with a $k$ dependent fluctuation of $\sigma \approx 0.05$. If we replace in the quantum multiple scattering series the amplitudes $t$ and $r$ by its FDA approximation [Eqs. (5.4) and (5.7)], the resulting unitarity limit at the same level of truncation, labeled as TQM-FDA, reaches value of 0.85 with $\sigma$ $\approx 0.05$. Turning now to the semiclassical theory based upon the FDA approximation including both classical and pseudopaths, we observe convergence as a function of a number of kinks $K$ included $(K \leqslant 10)$ towards the corresponding quantum unitarity limit. The only difference to the quantum result is the larger fluctuation $(\sigma \approx 0.15)$. This is in stark contrast to standard semiclassical approximations. The standard semiclassical result (without geometric path shadowing, $K=0$ ) exceeding the unitarity limit can be corrected for path shadowing denoted by $S^{S C A}[7,28]$. Longer paths $(\alpha, \beta)$ are shadowed by shorter paths $(\alpha / n, \beta / n)$ when $\alpha / n, \beta / n$ are integers for an integer $n$. Shadowing also removes geometric reflection at the open lead mouth (the "ghosts"). This correction, also shown in Fig. 7, reduces the transmission am-

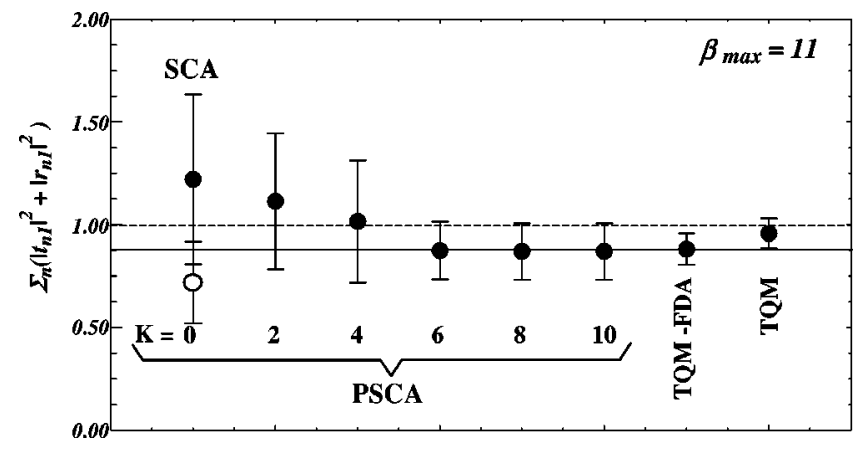

FIG. 7. Test of unitarity on different levels of semiclassical approximation to the rectangular billiard. Comparison between: quantum mechanical calculation with truncated multiple scattering expansion $\left(\beta_{\max }=11\right)$, TQM; quantum multiple scattering with FDA for diffractive amplitudes, TQM-FDA; pseudopath semiclassical approximation (PSCA) with $K=0, \ldots, 10 . K=0$ : standard SCA without path shadowing $\bigcirc$, with path shadowing $\bigcirc$. The billiard dimensions (see Fig. 1) are $D=L=\sqrt{4+\pi}$ and $d=0.25$. The average is performed over a $k$ window of $[\pi / d, 6 \pi / d]$.

plitude without restoring the unitarity. The present results illustrate that the semiclassical diffraction (or kink) expansion [Eq. (4.24)] converges towards the quantum calculation at the same level of input and truncation. It furthermore indicates that the largest residual error is caused by the FDA for $t$ and $r$ rather than from the semiclassical approximation to the paths sum itself, as indicated by the deterioration of the quantum multiple scattering series with FDA amplitudes. Employing diffraction approximations beyond the FDA such as the geometric theory of diffraction $[31,32]$ or the uniform theory of diffraction [33] would most likely result in further improvements.

Turning now to the path-length power spectrum for the transmission amplitude, $S_{11}=T_{11}$,

$$
P(\ell)=\left|\int d k e^{i k \ell} T_{11}(k)\right|^{2}
$$

the standard approximation $T_{11}(k) \approx S_{11}^{(0)}(k)$, [Eq. (4.24)] fails badly at large path length (Fig. 8). More precisely, while the peak positions which can be associated with classical paths and labeled by lattice vectors $\alpha, \beta$ agree well with the full quantum spectrum, the peak heights are overestimated up to one order of magnitude [Fig. 8(a)]. The quantum peak heights decrease exponentially while the standard semiclassical approximation exhibits only an inverse linear decay proportional to $\ell_{\alpha, \beta}^{-1}$. The present semiclassical theory that includes pseudopaths with a finite number of kinks, by contrast, reproduces the quantum path-length spectrum very well [Fig. 8(b)], even fine details at large $\ell$ are remarkably well reproduced. The decay at large $\ell$ becomes exponential rather than linear (Fig. 8c). This observation is the key to the understanding of the role pseudopaths play in quantum and semiclassical transport. The exponential suppression of the path-length spectrum at large $\ell$ is a consequence of the destructive interference of an exponentially proliferating set of 


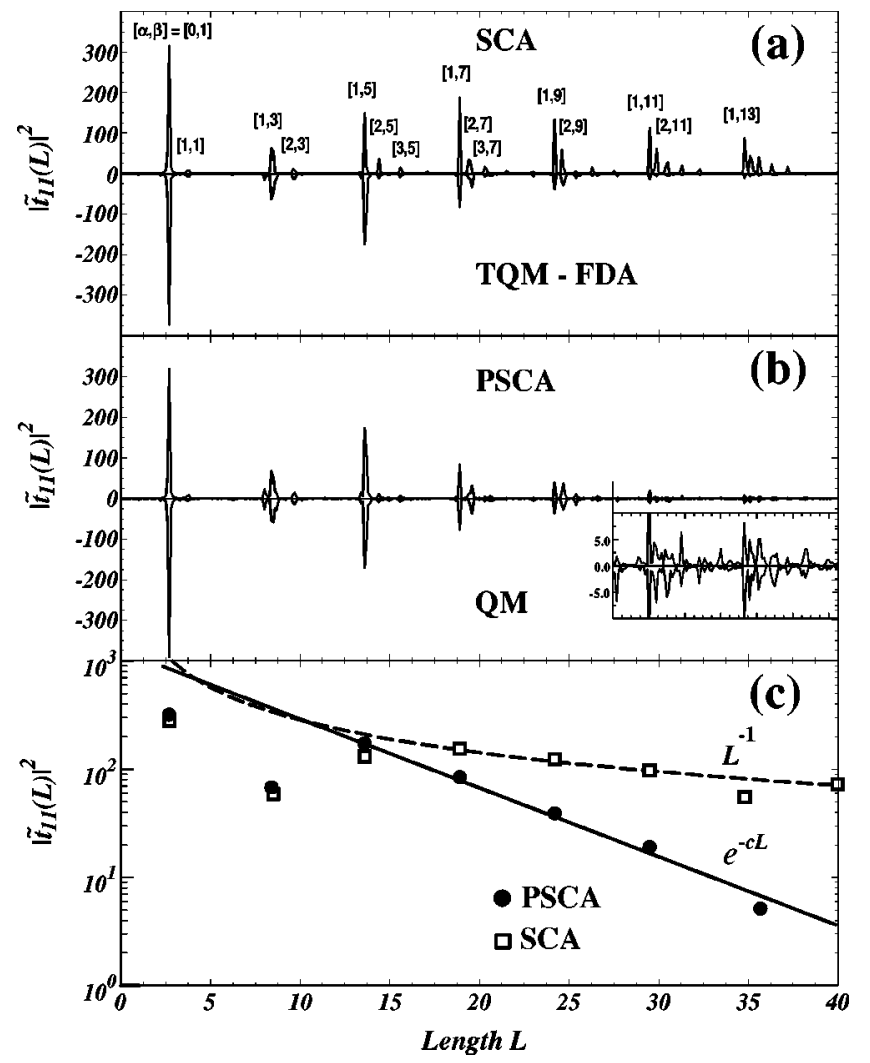

FIG. 8. (a) Comparison between standard SCA including FDA for emission and injection (Ref. [5]) and quantum spectrum (TQMFDA). (b) Comparison between pseudopath semiclassical approximation (PSCA) and quantum spectrum. (c) Exponential versus linear decay of path length spectrum in PSCA and standard SCA, respectively. Note that only the dominant peaks are plotted. Billiard dimensions and $k$ window as in Fig. 7.

pseudopaths. For each long classical zero-kink path with lattice vector $\alpha, \beta$ we find a large number $n$ of few-kink paths $\gamma$ with nearly the same length

$$
\ell_{\alpha, \beta}^{(\gamma)} \approx \sum_{K^{\prime}=1}^{K} \ell_{\Delta \alpha_{K^{\prime}} \Delta \beta_{K^{\prime}}}^{(\gamma)}, \quad \gamma=1, \cdots, n
$$

with

$$
\begin{aligned}
& \beta^{(\gamma)}=\sum_{K^{\prime}=1}^{K} \Delta \beta_{K^{\prime}}^{(\gamma)}, \\
& \alpha^{(\gamma)}=\sum_{K^{\prime}=1}^{K} \Delta \alpha_{K^{\prime}}^{(\gamma)},
\end{aligned}
$$

and whose Maslov indices differ from the zero-kink path. The exponential growth of pseudopaths with pseudorandomly varying phases assures the approximately exponential suppression of the contributions of long paths.

This observation also clears up another puzzle found in previous semiclassical calculations. The agreement between the semiclassical and the quantum path-length spectrum is much better for chaotic rather than for regular systems $[4,7,5,12,26]$ contrary to many other observables for which standard semiclassical approximation performs much better for regular rather than for chaotic systems. In chaotic systems, already classical paths exponentially proliferate as a function of the path length and can account for exponential suppression of large path lengths. Therefore, the lack of pseudopaths which also proliferate exponentially is less dramatically felt than in regular systems where the exponential proliferation of pseudo paths competes with only linear proliferation of classical paths.

\section{CONCLUSIONS}

We have presented a semiclassical theory for ballistic transport that goes beyond the standard semiclassical approximation by including an ascending order of diffractive scatterings in the interior of the ballistic cavity corresponding to certain classes of contributions in increasing order of $\hbar$. As the present formulation requires an additional and independent approximation for the elementary diffractive amplitudes for injection, emission, and internal reflection, inclusion of all contribution to a given order in $\hbar^{n}$ cannot be expected. Using the example of a rectangular billiard, we have shown numerically that an exponentially proliferating number of pseudopaths with diffractive kinks converges to the quantum multiple scattering series. In the rectangular billiard, the leads are the only sources of diffraction. For other geometries with concave walls or diffractive edges additional sources of diffraction are present. While the numerical result presented pertain to the rectangular billiard, we find numerical evidence that convergence towards quantum transport can be found for other geometries, specifically for the circle and the Bunimovich stadium [35]. We expect that our PSCA will also resolve other unresolved issues of semiclassical ballistic transport such as the problem of weak localization $[14,15]$ and the breakdown of symmetry of the autocorrelation function in reflection and transmission.

\section{ACKNOWLEDGMENTS}

Support by the FWF and by the FWF-SFB 016 is gratefully acknowledged.
[1] M.C. Gutzwiller, Chaos in Classical and Quantum Mechanics (Springer Verlag, New York, 1991), and reference therein.

[2] W. Miller, Adv. Chem. Phys. 25, 69 (1974).

[3] W.A. Lin, J.B. Delos, and R.V. Jensen, Chaos 3, 655 (1993).

[4] R.A. Jalabert, H.U. Baranger, and A.D. Stone, Phys. Rev. Lett.
65, 2442 (1990); H.U. Baranger, R.A. Jalabert, and A.D. Stone, Chaos 3, 665 (1993).

[5] H. Ishio and J. Burgdörfer, Phys. Rev. B 51, 2013 (1995).

[6] C.D. Schwieters, J.A. Alford, and J.B. Delos, Phys. Rev. B 54, 10652 (1996). 
[7] L. Wirtz, J.-Z. Tang, and J. Burgdörfer, Phys. Rev. B 56, 7589 (1997)

[8] L. Wirtz, J.-Z. Tang, and J. Burgdörfer, Phys. Rev. B 59, 2956 (1999).

[9] T. Blomquist and I.V. Zozoulenko, Phys. Rev. B 64, 195301 (2001); T. Blomquist and I.V. Zozoulenko, Phys. Scr., T T90, 37 (2001).

[10] R. Akis, D.K. Ferry, and J.P. Bird, Phys. Rev. Lett. 79, 123 (1997).

[11] E. Persson, I. Rotter, H.-J. Stöckmann, and M. Barth, Phys. Rev. Lett. 85, 2478 (2000).

[12] R.G. Nazmitdinov, K.N. Pichugin, I. Rotter, and P. Seba, Phys. Rev. E 64, 056214 (2001).

[13] R.G. Nazmitdinov, K.N. Pichugin, I. Rotter, and P. Seba, Phys. Rev. B 66, 085322 (2002).

[14] M. Hastings, A.D. Stone, and H. Baranger, Phys. Rev. B 50, 8230 (1994).

[15] A.D. Stone, Les Houches Session LXI (1994), edited by E. Akkermans et al. (North-Holland, Amsterdam, 1995), p. 329.

[16] L.A. Bunimovich, Funct. Anal. Appl. 8, 254 (1974).

[17] G. Benettin and J.M. Strelcyn, Phys. Rev. A 17, 773 (1978).

[18] E. Heller, Phys. Rev. Lett. 53, 1515 (1984).

[19] H. Friedrich, Theoretical Atomic Physics (Springer-Verlag, Berlin, 1998).

[20] M.V. Berry and K.E. Mount, Rep. Prog. Phys. 35, 315 (1972).

[21] J. Burgdörfer, C.O. Reinhold, J. Sternberg, and J. Wang, Phys. Rev. A 51, 1248 (1995).

[22] R. Landauer, IBM J. Res. Dev. 1, 223 (1957).
[23] See, e.g., A. Erdély, Asymptotic Expansions (Dover, New York, 1956), p. 51.

[24] R.P. Feynman and A. R. Hibbs, Quantum Mechanics and Path Integrals (MacGraw-Hill, New York, 1965).

[25] M.C. Gutzwiller in Chaos and Quantum Physics, edited by M.-J. Giannoni, A. Voros, and J. Zinn-Justin, (North-Holland, Amsterdam, 1991).

[26] S. Rotter, J.-Z. Tang, L. Wirtz, J. Trost, and J. Burgdörfer, Phys. Rev. B 62, 1950 (2000).

[27] See e.g., E. Merzbacher, Quantum Mechanics, 3rd ed. (Wien, New York, 1996).

[28] P. Pichaureau and R.A. Jalabert, Eur. Phys. J. B 9, 299 (1999).

[29] The Poisson sum formula (PSF) $\sum_{n=1}^{\infty} f(n)$ $=\Sigma_{l=-\infty}^{\infty} \int_{-\infty}^{\infty} f(\nu) e^{i 2 \pi l \nu} d \nu$ follows from the identity $\sum_{l=-\infty}^{\infty} e^{i 2 \pi l \nu}=\sum_{n=1 / 2}^{\infty} \delta(\nu-n)$. If $f(\nu)$ is assumed to be negligible for $\nu=0$, the PSF can be written as $\sum_{n=1}^{\infty} f(n)$ $=\frac{1}{2} \sum_{l=-\infty}^{\infty} \int_{-\infty}^{\infty} f(\nu) e^{i 2 \pi l \nu} d \nu$. For a summation over odd integers only, one obtains $\sum_{n=1}^{\infty} f(2 n+1)$ $=\frac{1}{4} \sum_{l=-\infty}^{\infty} \int_{-\infty}^{\infty} f(\nu) e^{i \pi l \nu} d \nu$.

[30] A. Sommerfeld, Vorlesungen über Theoretische Physik (Verlag H. Deutsch, Frankfurt, 1985), Vol. 5.

[31] J.B. Keller, J. Opt. Soc. Am. 52, 116 (1962).

[32] G. Vattay, J. Cserti, G. Palla, and G. Szálka, Chaos Solitons Fractals 8, 1031 (1997).

[33] M. Sieber, N. Pavloff, and C. Schmit, Phys. Rev. E 55, 2279 (1997).

[34] E. Bogomolny, Nonlinearity 13, 947 (2002).

[35] C. Stampfer, et al. (unpublished). 